\title{
Acknowledgment of Reviewers
}

\author{
Harold L. Drake, Editor in Chief
}

$\mathrm{M}$ aintaining the high quality of manuscripts published in AEM would not be possible without the efforts of our reviewers. Perceptive reviews are essential to AEM Editors as we strive to evaluate and improve submitted work. We are indebted to the members of our editorial board and would like to also publicly acknowledge the outstanding work of the many ad hoc reviewers we have worked with in the past year.

Jens Aamand

Usama R.

Abdelmohsen

Stephen T. Abedon

Maher Abou Hachem

Florence Abram

Nehal I. Abu-Lail

Martin Ackermann

Luis A. Actis

Gary R. Acuff

Jonathan M. Adams

Michael W. Adams

Nushin Aghajari

Brian M. Ahmer

Bertrand Aigle

Stuart R. Ainsworth

Michael D. Aitken

Robert A. Akins

Takuya Akiyama

Denise M. Akob

Ali Al-Ahmad

Mads Albertsen

Hans-Jørgen

Albrechtsen

Miguel Alcalde

Caitilyn Allen

Eric E. Allen

Grant D. Allen

Heather K. Allen

Lee-Ann H. Allen

Lisa Z. Allen

João R. Almeida

Rodrigo Almeida

Hal Alper

Andrew Alspaugh

Josef Altenbuchner

Miguel A. Alvarez

Lisa Alvarez-Cohen

Avelino Alvarez-

Ordóñez
R. G. Amachawadi

Linda A.

Amaral-Zettler

Carmen Amaro

Jan Amend

Achour Amiri

Jamila

Anba-Mondoloni

Anne J. Anderson

Burt Anderson

Gregory G. Anderson

Kirk E. Anderson

Michael Anderson

Björn Andersson

Maria Andersson

Thomas Andlid

Philippe Andre

Antoine Andremont

Cristina Andres-Barrao

Irini Angelidaki

Largus T. Angenent

S. Andreas Angermayr

Esther R. Angert

Clement Angkawidjaja

André Antunes

Rachael E. Antwis

Michael Apley

Jens Appel

Hiroyuki Arai

Takatoshi Arakawa

Wagner Araújo

Rosa M. Araujo Boira

Covadonga R. Arias

Beatrice Arico

Ludmilla Aristilde

Matthieu Arlat

Sandra K. Armstrong

Elizabeth Arnold

Nina Aro

Emma Aronson
Javier Arrizon

Terrance Arthur

Rebekka Artz

Bahareh Asadishad

Hiroshi Asakura

Vijay H. Aswani

Siavash Atashgahi

Mehmet R. Atilgan

Edward R. Atwill

Céline Audet

Manfred Auer

Marc D. Auffret

Jean-Christophe Augustin

Steven D. Aust

John W. Austin

Harriet Auty

Petri Auvinen

Joana Azeredo

Marli P. Azevedo

Susan J. Bach

Michael A. Bachman

David Backhouse

Jin-Woo Bae

Michael Bagdasarian

Ying Bai

Andy Bailey

Aurélien Bailly

Xavier Bailly

Katherine H. Baker

Matthew G. Bakker

Laura Baldo

Carl Balibar

Nicholas R. Ballor

Emily P. Balskus

David A. Baltrus

Richard H. Baltz

Charles W. Bamforth

Fernando Baquero

Jozsef Baranyi
Mariette Barbier

Alan G. Barbour

Jose E. Barboza-Corona

Tamar Barkay

Adam P. Barker

Andrew C. Barnes

Brett M. Barney

Riccardo Baroncelli

Nicolas Barraud

Josefina Barrera-Cortés

Rebecca Bart

Hazel A. Barton

Larry Barton

Mirko Basen

Gilles J. Basset

Ashley Bateman

Antonio Battisti

Dennis Baumgardner

Joaquin Bautista Gallego

Edward A. Bayer

Kenneth W. Bayles

Matthew Baylis

Navid Bazghaleh

Dennis A. Bazylinski

Denise M. Bazzolli

Gwyn A. Beattie

Andreas Bechthold

Jeffrey M. Becker

Katie Becklin

Eric D. Becraft

Malgorzata Bednarska

Steven V. Beer

Sebastian Behrends

Chase Beisel

Asim K. Bej

Robert Belas

Lisa K. Belden

Álvaro Belenguer

Harry R. Beller
Keaton M. Belli

Gaël Belliot

Michael J. Benedik

Frank R. Bengelsdorf

José A. Bengoechea

Joan W. Bennett

Gabriele Berg

Ivan Berg

Odile Berge

John A. Berges

Maria Bergsma-Vlami

Randy M. Berka

Bert Berla

Luis G.

Bermúdez-Humarán Jean-François

Bernarded

Rizlan Bernier-Latmani

Jean-Guy Berrin

David Berry

Anthony D. Bertagnolli

Catherine Berthomieu

Ralph Bertram

Yvan Bettarel

Larry R. Beuchat

Lothar Beutin

Catherine Bevier

Haluk Beyenal

Deepak Bhatnagar

Devaki Bhaya

Arun K. Bhunia

Mervyn J. Bibb

Jennifer F. Biddle

Michael J. Bidochka

Gabriele Bierbaum

Elisabeth Biesta-Peters

Dawn Bignell

Elisabeth M. Bik

Nicole Billings

Craig Billington 
John Bingham

Matthew J. Binnicker

Elena Biosca

Patrick J. Blackall

Anicet R. Blanch

Jorge Blanco

Lars Blank

Rafael Blasco

Michael Blaut

Bastian Blombach

Paul Blum

Robert M. Blumenthal

Sara E.

Blumer-Schuette

Thomas A. Bobik

Lynne Boddy

Helge B. Bode

Alexandria B. Boehm

Zuzana Bohrerova

Nicholas A. Bokulich

Annette Bollmann

Philip L. Bond

Gregory Bonito

Violaine Bonnefoy

Adam J. Book

Kenneth Boorom

Seth Bordenstein

Anthony Borneman

James Borneman

Irina Borodina

Abhijeet P. Borole

Rafael Borras Salvador

Giorgio Borreani

Rainer Borriss

Leticia Botella

Michael Bott

David G. Bourne

Bob Bourret

John P. Bowman

John D. Boyce

Eric Boyd

Ethna F. Boyd

Damien Brady

Olivier Braissant

Luca Brambilla

Rafael Brandão Varella

Adriano Brandelli

Roderich Brandsch

Kristian Brandt

Mary E. Brandt

Guy Branlant

Susan L. Brantley

Suzanna L. Brauer

Annett Braune

Alejandra Bravo

Byron F.

Brehm-Stecher

Phil Bremer

Michael R. Brent

Jean-François Briand
Romain Briandet

Christopher J. Brigham

Thorsten Brinkhoff

Shaun R. Brinsmade

Nichole A. Broderick

Lone Brøndsted

John Brooks

Lubov Brovko

Daniel R. Brown

Pamela J. Brown

Steven D. Brown

Neil C. Bruce

Christian Bruckner

Andreas Brune

Roman Brunecky

Vincent M. Bruno

José M. Bruno-Bárcena

Thomas Bruns

Carolyn Buchwald

Timothy D. Bugg

Bruno Bühler

George Bullerjahn

David Bundle

Andre G. Buret

David Burke

Sandra H. Burnett

Cara C. Burns

Lori L. Burrows

Sara A. Burt

Milos Busarcevic

Kathryn Bushley

Patrick Butaye

Ed T. Buurman

Primitivo Caballero

Ricardo M. Cabrera

Michael W. Calfee

Douglas F. Call

Gloria Caminal

Douglas Campbell

Anne Camper

Pietro Canepari

Jason Cantera

Guojie Cao

Min Cao

Vittorio Capozzi

Gianluigi Cardinali

Frederic Carlin

John H. Carlson

David Carmena

Francisco Carrau

Manuel Carrilho

Catherine D. Carrillo

Jeffrey W. Cary

Lisa Casanova

Jennifer L. Cashdollar

Bryan J. Cassone

Mariana Castanheira

Daniel Castillo

Santiago

Castillo-Ramírez
Javier Castro-Rosas

Artur Cavaco-Paulo

Jean-François Cavin

Natalia Cernicchiaro

Francisco J. J.

Cervantes

Romy Chakraborty

Gemma Chaloner

Clara Chan

James W. Chan

Michael Chandler

Abhirosh Chandran

Matthew Chang

Yung-Fu Chang

Matthew R. Chapman

Steve J. Charette

Daniel Charlier

John Chaston

Keith Chater

Subhadeep Chatterjee

Vishnu Chaturvedi

Keya Chaudhuri

Luis E. Chávez de Paz

Guo-Qiang Chen

Jian Chen

Liang Chen

Rachel R. Chen

Shawn Chen

Yihua Chen

Yun Chen

Zhao Chen

Ying Cheng

Chien-Shun Chiou

Jean-Marc Chobert

Byung-Kwon Choi

Bruno B. Chomel

Graham Christie

Peter J. Christie

Konstantin Chumakov

Matthew Church

Maurizio Ciani

Matheus A. Cipriano

Jon Clardy

Katherine W. Clark

Richard B. Clark

Christopher Clarke

David J. Clarke

Keith Clay

Nicholas Clipson

Sarah A. Clock

Paul A. Cobine

Tom Coenye

Maureen Coleman

Nicholas V. Coleman

Michael T. Collins

Duncan Colquhoun

André M. Comeau

Laurie E. Comstock

Ralf Conrad

Lorenza Conterno
Gregory M. Cook

Perran Cook

Terrance G. Cooper

Mary Corcoran

Pierre Cornelis

Cynthia N. Cornelissen

Maria G. Corradini

Jennifer Cory

Adele Costabile

Peter D. Countway

Mathieu Coureuil

Michael Cox

Russell Cox

Aurélie Crabbé

Irena Creed

James M. Cregg

Melissa A. Cregger

Carole Creuzenet

Theresa Cromeans

Jo A. Crouch

Pedro Crous

Byron C. Crump

Jaime Cubero

Zhongli Cui

Alexander I. Culley

Leonardo Curatti

Cameron R. Currie

Chris Curtin

Wayne R. Curtis

Leah Cuthbertson

Heribert Cypionka

Daniele Daffonchio

Stefania Daghino

Denise Dagnino

Christiane Dahl

Xin Dai

Colin Dale

Paw Dalgaard

David Dance

Antoine Danchin

Ajai A. Dandekar

Geoffrey Daniel

Chuck Daniels

Eric Dannaoui

Guo Daoyi

Jean-Marc G. Daran

Annie Dary-Mourot

Gregory A. Dasch

Alexandre DaSilva

Gabriela J. da Silva

Atin R. Datta

Günther Daum

Julian E. Davies

Margaret A. Davis

Meghan F. Davis

Angus L. Dawe

Maria De Angelis

Laurent Debarbieux

Dirk de Beer

Gustavo M. de Billerbeck

Irene de Bruijn

Ellen Decaestecker

Alessandra De Cesare

Tom Defoirdt

Patrick Degnan

Leo H. de Graaff

Stefan de Kok

Leonardo De La Fuente

César de la

Fuente-Núñez

Maria Angeles de la

Torre-Ruiz

Frédéric Delbac

Antonio Dell'Anno

Edward F. DeLong

Susan K. De Long

Victor de Lorenzo

Francis de los Reyes

Maurizio Del Poeta

Frank Delvigne

Pieter De Maayer

Arnold L. Demain

Jill E. Demers

Erick Denamur

Xiangyu Deng

Ye Deng

Uwe Deppenmeier

Sylvie Dequin

Andrew P. Desbois

David DeShazer

Tom Desmet

Christelle Desnues

Evandro L. de Souza

Corrella S. Detweiler

Josef Deutscher

Aurèlie Deveau

Frank Devlieghere

Paul De Vos

Willem M. de Vos

Bert Devriendt

Ronald P. de Vries

Luc De Vuyst

Rosemarie De Weirdt

Eric Deziel

Wim DHaeze

Abhishek S. Dhoble

Eduardo Díaz

Dulce M. 
Alan A. DiSpirito

Elke Dittmann

Katharina Dittmar

Maria Diuk-Wasser

Benoit Divol

Dylan Dodd

William T. Doerrler

Yohei Doi

Marguerite

Dols-Lafargue

Michael M. Domach

Stefano Donadio

Niles P. Donegan

Hailiang Dong

Maureen J. Donlin

Siobhan Dorai-Raj

James R. Doroghazi

David W. Dorward

François P. Douillard

Maxwell Dow

Sean Doyle

Shaynoor Dramsi

Djamel Drider

Barbara Drigo

Adam Driks

Zhao-jun Duan

Erik Dubberke

Eric Duchaud

Geraldine Duffy

Eric Dugat-Bony

Erwin Duizer

Aurélien Dumètre

Marc G. Dumont

John Dunbar

Peter Dunfield

Lysiane Duniere

Paul Dunlap

Anne K. Dunn

Robert Duran

Peter Dürre

Belma Durupinar

Maret du Toit

Myke Dyall-Smith

Sonya Dyhrman

Gary A. Dykes

Dariusz Dziga

Leo Eberl

Carrie Eckert

Ulrich Eckhard

Shigetoshi Eda

Winfried Edelmann

Mira Edgerton

Thomas D. Edlind

Kyle Edwards

Marc A. Edwards

Simon Edwards

Uta Effmert

Armin Ehrenreich

Matthias Ehrmann

Jerry Eichler
Jessica J. Eichmiller

Stephanie A. Eichorst

Thilo Eickhorst

Alexander Eiler

Dwayne Elias

Andrew C. Eliot

James G. Elkins

Craig D. Ellermeier

Kathryn T. Elliott

Matthew L. Ellison

John Elphinstone

Lindsay D. Eltis

Joanne B. Emerson

Philipp Engel

Francis Enjalbert

Saïd Ennahar

Scott Ensign

K.-D. Entian

Scott E. Erdman

Marilyn Erickson

Leonardo Erijman

Peter B. Ernst

Adelfo Escalante

Baltasar Escriche

Eduardo A. Espeso

Guadalupe Espín

A. V. Espinel-Ingroff

Manuel Espinosa

Manuel Espinosa-Urgel

Ana C. Esteves

Katharina F. Ettwig

James Euan

Oystein Evensen

Thaddeus Ezeji

Patrick Fach

Joana S. Falcão

Peter Falck

Linda Falgenhauer

Knut Falk

Yanhua Fan

Zhiliang Fan

Francesca Fanelli

Ferric C. Fang

Francesco Faretra

Andreas H. Farnleitner

Michael Fasseas

Craig Faulds

Guido Favia

Justin Fay

Michael J. Federle

Claus Felby

Jie Feng

Donna E. Fennell

Timothy Ferdelman

Dwight D. Ferguson

Heriberto Fernandez

Roberto

Fernandez-Lafuente

María

Fernández-Lobato
Juan Ferré

Patricia Ferreira Neila

Manuel Ferrer

J. G. Ferry

Andrea T. Fessler

Susanne Fetzner

Paul D. Fey

Thomas A. Ficht

David Fields

Matthew W. Fields

María J. Figueras

Sabine Fillinger

Turlough M. Finan

Kevin T. Finneran

Lucia Fiore

Carol L. Fischer

Reinhard Fischer

Sonia E. Fischer

Robert F. Fisher

Benjamin Fitzpatrick

John Flannery

Graham Fleet

Andre Fleissner

Enrique Flores

Michael Flythe

Christiane Forestier

Steven A. Forst

Jan Förster

Patrick Forterre

Louis-Charles Fortier

Jamie S. Foster

Jeffrey T. Foster

Kevin Foster

Brian G. Fox

James G. Fox

Bart A. Fraaije

Bernardo Franco

Jean M. François

Karen Frank

Kristi L. Frank

Gad Frankel

Eelco Franz

Pina M. Fratamico

Jim K. Fredrickson

Maria

Fredriksson-Ahomaa

David L. Freedman

Dorte Frees

Remo Freimann

Michael Freitag

Nancy E. Freitag

Alexander D. Frey

Anika Friese

Jens C. Frisvad

Brett A. Froelich

Åsa Frostegård

Bernhard M. Fuchs

Georg Fuchs

John A. Fuerst

Takashi Fujikawa
Tomoko Fujiyuki

Satoru Fukiya

Toshiaki Fukui

Matthew S. Fullmer

Barbara Funnell

Vincenzina Fusco

Shinya Fushinobu

Taiki Futagami

Hiroyuki Futamata

Chris Gaby

Geoffrey M. Gadd

Daniel J. Gage

Pawel Gajer

Alexios Galanis

Cesira L. Galeotti

Antoine Galiana

Luca Galluzzi

David L. Gally

Giovanni Ganendra

Phil Ganter

Haichun Gao

Javier Garaizar

Paolina Garbeva

Pilar García

Cristina García-Aljaro

Carlos García-Estrada

Ana L. García-Perez

Luiz Gustavo A.

Gardinassi

Rafael A. Garduño

Daniel Garrido

Daniel R. Garza

H. R. Gaskins

Josep M. Gasol

Rebecca J. Gast

Eric Gaucher

Purushottam V. Gawande

Timothy G. Geary

Wei Gehong

Otto Geiger

Rolf Geisen

Terry J. Gentry

Dimitris Georgellis

Nicole M. Gerardo

Charles P. Gerba

Robin Gerlach

Jonathan Gershenzon

Kati Geszvain

Murad Ghanim

Jean-Marc Ghigo

Federica Giacometti

Maria S. Gião

Penelope S. Gibbs

Brian Gibson

Lisa Gieg

Adriana J. Gielbert

Stefan Giesen

Jason J. Gill

Philippe Gilot

Matthew Ginder-Vogel

Stephen J. Giovannoni

Giorgio Giraffa

Peter R. Girguis

Douglas P. Gladue

Frank Gleason

Anthony Glenn

Matthew R. Goddard

André Goehler

Darla M. Goeres

Michael Goettfert

Shana Goffredi

Digambar V. Gokhale

Piotr Golec

Carlos C. Goller

Peter N. Golyshin

Tânia A. Gomes

Maria Gomes-Solecki

Isabel Gómez

Elena Gonella

Graciela Gonzalez-Gil

Barry Goodell

David M. Gordon

Yuval Gottlieb

Arun Goyal

Stefan Graessle

Sara E. Gragg

Corinne Grangette

Irene R. Grant

Per E. Granum

Brian Gratwicke

Paul R. Grbin

Susannah Green Tringe

Kelvin B. Gregory

Elizabeth Grice

Christian Griebler

David E. Griffith

Gareth W. Griffith

John F. Griffith

Magdalena Grifoll

Dennis Grogan

Elisabeth Grohmann

Dennis C. Gross

Ariel Grostern

Stephen F. Grove

Marvin J. Grubman

Amy M. Grunden

Jean Guard

Luca Guardabassi 


\begin{tabular}{|c|c|c|c|c|}
\hline Adam M. Guss & Burkhard A. Hense & Sharon A. Huws & Yong-Su Jin & Junichi Kato \\
\hline Bertolt Gust & Craig Herbold & Cheng-An Hwang & Juan T. Jofre & Masashi Kato \\
\hline Santiago Gutiérrez & Mark T. Hernandez & Yuki Ichinose & G. S. Johannessen & Souichiro Kato \\
\hline Tony Gutierrez & Pablo E. Hernández & Yuichiro Iida & Per Johansson & Leonard Katz \\
\hline Charles N. Haas & Carmen Sara & Sanja Ilic & Sophia Johler & Michael S. Kay \\
\hline Hiroshi Habe & Hernández-Rodriguez & Jeongdae Im & Reimar Johne & Jay D. Keasling \\
\hline Timothy J. Hackmann & Joshua Herr & Wan-Taek Im & David Johnson & David H. Keating \\
\hline Johannes H. Hackstein & Alfredo H. & Tomoya Imai & David R. Johnson & Shivaramu Keelara \\
\hline Marisa Haenni & Herrera-Estrella & Kenji Inagaki & Eric A. Johnson & Veerappa \\
\hline Freddy Haesebrouck & Jean L. Herrmann & Karl J. Indest & Hope A. Johnson & Nancy Keller \\
\hline Martin Hagemann & Martina Herrmann & Hanne Ingmer & Roger P. Johnson & Christina A. Kellogg \\
\hline Max M. Häggblom & Christian Hertweck & Kengo Inoue & Timothy J. Johnson & Steven L. Kelly \\
\hline Ji-Sook Hahn & Wolfgang Hess & Yoshiharu Inoue & Andrew W. Johnston & William J. Kelly \\
\hline Matthias Hahn & Kim Heylen & William P. Inskeep & Alexander Johs & Frank Kempken \\
\hline Feth el Zahar Haichar & David S. Hibbett & Panagiotis Ioannidis & Jeffrey B. Jones & Charles M. Kenerley \\
\hline Patrick Hallenbeck & Friederike Hilbert & Richard E. Isaacson & Jessica Jones & Servé Kengen \\
\hline Jong H. Ham & Kristiina Hildén & Suzanne L. Ishaq & Patrik Jones & Cheryl A. Kerfeld \\
\hline Trinity L. Hamilton & Kristina Hillesland & Kazuyuki Ishihara & Ryan T. Jones & Philip J. Kersten \\
\hline Brian K. Hammer & Sarah Hind & Satoshi Ishii & Stuart Jones & Tajalli Keshavarz \\
\hline Karin Hammer & Shin-ichi Hirano & Michihiro Ito & Tineke Jones & James Ketudat-Cairns \\
\hline John Hammond & Ann M. Hirsch & Angela Ivask & Wilfried Jonkers & Nemat O. Keyhani \\
\hline Kap-Hoon Han & Ryan M. Hoffman & Kazuhiro Iwashita & Kieran Jordan & Madona Khoury \\
\hline Sukkyun Han & Ary A. Hoffmann & Javier Izquierdo & Milko Jorquera & Cezar M. Khursigara \\
\hline Yejun Han & Kirsten Hofmockel & Elina Jääskeläinen & Elsa Jourdain & Morten C. \\
\hline Marja-Liisa Hänninen & Martin Hofrichter & Charlene R. Jackson & Alexandre Jousset & Kielland-Brandt \\
\hline Allison Hansen & Thomas B. Hofstetter & Megan Jacob & Fabien Joux & Ronald P. Kiene \\
\hline Brian Hanzelka & Deborah Hogan & Karin Jacobs & Kou-San Ju & Yoshitomo Kikuchi \\
\hline Lingyun Hao & Jennifer N. Hogan & Deborah Jacobs-Sera & Andrew Juhl & Nabil Killiny \\
\hline Ivan Hapala & Lone Hoj & Ursula Jakob & Vincent Juillard & Dennis H. Kim \\
\hline Sajeet Haridas & James F. Holden & Pawel Janczyk & Mattijs K. Julsing & Diane Y. Kim \\
\hline Falk Harnisch & James T. Hollibaugh & Stefan Janecek & Robert Junker & Jae-Han Kim \\
\hline Jonathan Harris & Emily B. Hollister & Christoph Jans & Heli Juottonen & Pil Kim \\
\hline Linda Harris & Pascal Hols & Jan Jansa & Klaus Jürgens & Sang Y. Kim \\
\hline Joe Harrison & Jiann-Ruey Hong & Dick Janssen & Edouard Jurkevitch & Gary M. King \\
\hline Robert L. Harrison & Qing Hong & Robert Janulczyk & Mirjam Kaestli & David H. Kingsley \\
\hline John S. Hartung & Yaoqin Hong & Laura R. Jarboe & Justin Kaffenberger & Robert A. Kingsley \\
\hline Caroline S. Harwood & Kelli Hoover & Jennifer A. Jay & Amy Kahler & Nardy Kip \\
\hline Colin Harwood & Petr Horák & Lee-Ann Jaykus & Jörn Kalinowski & David L. Kirchman \\
\hline Roland Hatzenpichler & Katsutoshi Hori & Michele T. Jay-Russell & Martin L. Kalmokoff & Corby Kistler \\
\hline Aviaja Hauptmann & Peter Hortschansky & Yeon Ho Je & Martin Kaltenpoth & Hiroko K. Kitamoto \\
\hline Robert P. Hausinger & Hisashi Hoshida & Thomas W. Jeffries & Marina Kalyuzhnaya & Motomitsu Kitaoka \\
\hline Susanne Häußler & Kurt Houf & Johannes A. Jehle & Yoichi Kamagata & Çiğdem Kıvılcımdan \\
\hline Anthony G. Hay & Adina C. Howe & Michael B. Jenkins & Jinjun Kan & Moral \\
\hline Finbarr Hayes & Yi-huang Hsueh & Howard F. Jenkinson & Edna S. Kaneshiro & Stephan Klähn \\
\hline Ronen Hazan & Yan $\mathrm{Hu}$ & Francis E. Jenney & Hyun Ah Kang & Jonathan Klassen \\
\hline Jian $\mathrm{He}$ & Lihan Huang & Anders Jensen & Masashi Kanki & Günter Klein \\
\hline Jianzhong $\mathrm{He}$ & Qiaoyun Huang & Dane Jensen & Cheng Kao & Sara Kleindienst \\
\hline Sabrina Hedrich & Ying Huang & Paul R. Jensen & Katy C. Kao & Athol V. Klieve \\
\hline Nagendra R. Hegde & Julie A. Huber & Peter R. Jensen & T. J. Kappock & Kirstine Klitgaard \\
\hline Annamari & Casey R. Hubert & Susan E. Jensen & Levente Karaffa & Martin G. Klotz \\
\hline Heikinheimo & Laura A. Hug & Mollie W. Jewett & Petros C. Karakousis & Jochen Klumpp \\
\hline Thorsten Heinekamp & Jonathan G. Hughes & Pingsheng Ji & Musafiri Karama & Stephen Knabel \\
\hline Jürgen J. Heinisch & Jenni Hultman & Cheng-Ying Jiang & David M. Karl & Claudia Knief \\
\hline Robert A. Heinzen & Paul Humphreys & Jiandong Jiang & Petr Karlovsky & Olaf Kniemeyer \\
\hline Elmar Heinzle & Martha Hunter & Mengxi Jiang & Ida Karlsson & Donald Kobayashi \\
\hline Hermann J. Heipieper & Neil Hunter & Nannan Jiang & Mohamed Karmali & Tetsuo Kobayashi \\
\hline Ferdi L. Hellweger & Wayne B. Hunter & Weihong Jiang & Kaja Kasemets & Michal Koblizek \\
\hline Richard F. Helm & Anwar Huq & Yongqin Jiao & Kazem Kashefi & Matthew D. Koci \\
\hline Thierry Hennebelle & Mark R. Hurst & Miao Jin & Anne-Kristin Kaster & Robin Köck \\
\hline Celine Henry & Claudia Husseneder & Yan Jin & Sophia Kathariou & Ralf Koebnik \\
\hline
\end{tabular}




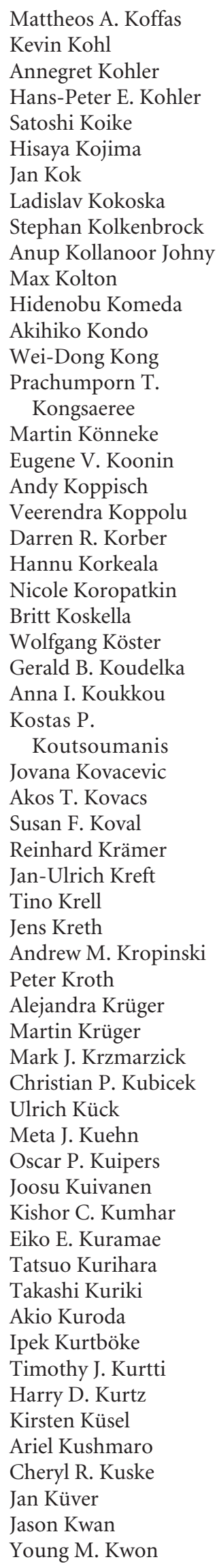

Jessica Labonte

Matthias Labrenz

Yannick Labreuche

Steve Labrie

Jan-Wilm Lackmann

Victor Ladero

Olga M. Lage

Katie Laird

Todd LaJeunesse

Tommy T. Lam

Regina Lamendella

Alicia Lammerts van Bueren

Christian Landry

Andrew S. Lang

Laurie LaPat-Polasko

Marta Laranjo

Andrea Larsen

Ray A. Larsen

Bernard La Scola

Xavier Latour

Christian Lauber

Christopher Lawrence

Carlo Lazado

Beth Lazazzera

Anna Lazzaro

Sarah Lebeer

Sarah Lebeis

Jean Guy LeBlanc

François Lebreton

Franziska Lederer

Alvin Lee

Dong-Yup Lee

Jinwon Lee

Margie D. Lee

Natuschka M. Lee

Patrick Lee

Sang Y. Lee

W. Theodore Lee

Younghoon Lee

Mary-Cathrine Leewis

Christopher T. Lefèvre

Laura G. Leff

Matic Legiša

Françoise S. Le

Guyader

James A. Leigh

John A. Leigh

Jorge H. Leitão

Christophe Le May

Michael J. Lemke

Hervé Le Moual

Oliver Lenz

Susan R. Leonard

Josefina León-Félix

Jacques Le Pendu

John F. Leslie

Daniel J. Lessner

Andrey V. Letarov

Mark A. Lever
Jean-Pierre Levraud

Karen Levy

Alan Lewitus

Alex Li

DeWei Li

Fu-Li Li

Jianrong Li

Liande Li

Qian Li

Robert W. Li

Shengying Li

Wen-Jun Li

Xiangzhen Li

James C. Liao

Wei Liao

Xinggang Liao

Stephen J. Libby

Tine R. Licht

Gunnar Liden

Mary E. Lidstrom

Michael Lienemann

Christiane Liers

Mark R. Liles

Erik Lilleskov

Direk

Limmathurotsakul

M. Carmen Limón

Jun Lin

Tsair-Fuh Lin

Yuheng Lin

Z. L. Lin

Björn Lindahl

Pia Lindberg

Celeste C. Linde

Markus B. Linder

Tomas Linder

Nic D. Lindley

H. D. Alan Lindquist

Diane Lindsay

Eva Lindström

Miia Lindström

Robert Linhardt

Philipp Lins

John E. Linz

Erin K. Lipp

John D. Lippolis

Wayne Litaker

John H. Litchfield

Chang-Jun Liu

Lei Liu

Liming Liu

Weifeng Liu

Ya-jun Liu

Yiming Liu

Zhi-Pei Liu

Per O. Ljungdahl

David Lloyd

Thomas P. Loch

Shawn R. Lockhart

Jean Lodge
Charlotta Löfström

Antonio Logrieco

Katherine S. Long

Aline Lonvaud

Rolf Lood

Juan Loor

Lyliam Loperena

Paloma Lopez

Graciela L. Lorca

Jeffrey M. Lorch

Jennifer J. Loros

Chunbo Lou

Ed J. Louis

Derek R. Lovley

Anhuai Lu

Shien $\mathrm{Lu}$

Xiaonan Lu

Xuefeng Lu

Petra Luber

Patrick M. Lucas

Catharina H. Lüdeke

Brandon E. Luedtke

Camile Lugarini

Joen Luirink

Michelle R. Lum

Taina K. Lundell

Gang Luo

Xin M. Luo

Yuanming Luo

Lee R. Lynd

Inna Lysnyansky

Kesen Ma

Donna M. MacCallum

Barbara J. MacGregor

Milton Maciel

Roderick I. Mackie

Jean-Yves Madec

Tatsuya Maeda

Timothy Magnuson

Biswanath Mahanty

Jacques G. Mainil

Tim Maisch

Rex R. Malmstrom

Renato P. Maluta

Nikhil S. Malvankar

Scott R. Manalis

Charles Manceau

Mike Manefield

John M. Mansfield

Leonie Marang

Jose F. Marcos

Gregory T. Marczynski

Julia A. Maresca

Abelardo Margolles

Sonia Marín

Julia Marín-Navarro

Robert Marra

Terence L. Marsh

Ian P. Marshall
Willm

Martens-Habbena

Javier Martin

Juan F. Martín

Mark O. Martin

Nancy L. Martin

Vincent Martin

José L. Martínez

Juan J. Martinez

Santiago

Martínez-Calvillo

Paloma

Martínez-Rodríguez

Sergio

Martínez-Rodríguez

Esperanza

Martínez-Romero

Ludmila Martínková

Marta Martins

Vincent G. Martinson

Jan Martinussen

Adam Martiny

Jennifer Martiny

Florentine Marx

Albert Mas

Isabelle

Masneuf-Pomarède

Olivia Mason

Robert P. Mason

Matthew D.

Mastropaolo

Christopher K.

Mathews

Miguel A. Matilla

Kazunobu Matsushita

Shigenobu Matsuzaki

Diethard Mattanovich

Andrea Mattevi

Hans C. Matthijs

Heather Maughan

Julie A. Maupin-Furlow

Max Maurin

Harold D. May

Tim A. McAllister

Olivia McAuliffe

Mark J. McBride

Shonna M. McBride

Alan J. McCarthy

Peter J. McCarthy

Bruce A. McClane

Siobhan McClean

Michael McConnell

Joseph R. McCormick

Susan P. McCormick

Diane McDougald

David A. McDowell

Neil R. McEwan

Quinn S. McFrederick

Terry McGenity

Elizabeth A. McGraw 


\begin{tabular}{|c|c|c|c|c|}
\hline Bradford S. McGwire & Rachael M. & Kristian Fog Nielsen & Jörg Overmann & Johann Pitout \\
\hline Ailsa McLean & Morgan-Kiss & Per H. Nielsen & Yasuhiro Ozeki & Dipti W. Pitta \\
\hline Katherine McMahon & Peter Morin & Timo T. Nieminen & Yakov Pachepsky & Mariana Piuri \\
\hline David S. McNabb & Yuki Morono & Dietrich H. Nies & Lokesh Padhye & Pascal Piveteau \\
\hline Paul McSweeney & Jeff Morrell & Ivonne Nijenhuis & Franco Pagotto & Gregory V. Plano \\
\hline David Mead & Cindy E. Morris & Angela M. Nilius & Subhamoy Pal & Thomas G. Platt \\
\hline John C. Meeks & Dearbháile Morris & Hiromi Nishida & Grazyna Palamarczyk & Jürgen Pleiss \\
\hline Friedhelm Meinhardt & J. Jeffrey Morris & Kunihiko Nishino & Oleg Paliy & Ana Plemenitas \\
\hline Luis Mejia & Robert M. Morris & Rachel T. Noble & Kelli L. Palmer & Caroline M. Plugge \\
\hline Nadja R. Melo & Kathleen M. Morrow & Justin R. Nodwell & Mitchell Palmer & Jacqueline A. \\
\hline Stephen B. Melville & Kathy Morrow & Daniel R. Noguera & Robert J. Palmer & Plumbridge \\
\hline Beatriz Méndez & Joachim Morschhäuser & Kenneth M. Noll & Timothy Palzkill & István Pócsi \\
\hline Artemio & Gloria Mosquera & Joakim Norbeck & Daniel G. Panaccione & Stefanie Poeggeler \\
\hline Mendoza-Mendoza & Xiaozhen Mou & Benjamin Normark & Efstathia Papafragkou & Ansgar Poetsch \\
\hline Fangang Meng & Rebecca Mueller & Steven J. Norris & Eleftherios T. & Laurent Poirel \\
\hline Yvette Merga & Richard W. Muirhead & Jeanette M. Norton & Papoutsakis & Isabelle Poirier \\
\hline Max Mergeay & Amit Mukherjee & Paige J. Novak & Nádia S. Parachin & Lubos Polerecky \\
\hline Guillaume Meric & Aindrila & Linda Nunan & Gyungsoon Park & Andrea Polle \\
\hline D. S. Merrell & Mukhopadhyay & Leonard Nunney & Joonhong Park & Shawn W. Polson \\
\hline Steven R. Meshnick & Rudolf Müller & George-John E. Nychas & Matthew A. Parker & Holly Popham \\
\hline João R. Mesquita & Adam Mumford & Spencer V. Nyholm & Jerry M. Parks & Michel-Robert Popoff \\
\hline Mikko Metsä-Ketelä & Christopher Mundt & Brian B. Oakley & Matthew R. Parsek & Milka Popova \\
\hline Philip Meuleman & Alsayadi Muneer & Ingrid Obernosterer & Laila Partida-Martinez & Jürgen Popp \\
\hline Dik J. Mevius & Colin B. Munn & Conor O’Byrne & Javier Pascual & Matthew C. Posewitz \\
\hline Filip Meysmann & Rosario Muñoz & Francisco M. Ochoa- & Luciane M. P. Passaglia & Michael Poulsen \\
\hline Herbert Michlmayr & Kenan C. Murphy & & Volkmar Passoth & Christine Pourcel \\
\hline Peter Miethe & Marc Mussmann & Kevin P. O’Connell & Jitendra Patel & Radha Prasanna \\
\hline Aaron W. Miller & Phillip R. Myer & Páraic Ó Cuiv & Timothy C. Paulitz & James F. Preston \\
\hline Patti J. Miller & T. G. Nagaraja & Yasuhiro Oda & Helene M. M. Paxton & Joanne Preston \\
\hline Samuel I. Miller & Keiji Nakamura & Kerry O’Donnell & Gary A. Payne & G. Dean Price \\
\hline F. Chris Minion & Shugo Nakamura & Jun Ogawa & Michael W. Peck & Lance B. Price \\
\hline Samuel Minot & Michiko M. Nakano & Marco R. Oggioni & Kirsten Pelz-Stelinski & Peter Proksch \\
\hline Nigel P. Minton & Yutaka Nakashimada & Andrew Ogram & Joseph T. Penrod & Amy Pruden \\
\hline Babur S. Mirza & Shigetou Namba & Kumiko Oguma & C. Ryan Penton & Olivier Pruvost \\
\hline Birgit Mitter & Franz Narberhaus & Satoshi Okabe & Lily Pereg & Carla Pruzzo \\
\hline Takayuki Miura & Uwe Nehls & Yaacov Okon & Mirjam Perner & Natalie A. Prystajecky \\
\hline Masatoshi Miyakoshi & David E. Nelson & Hidetoshi Okuyama & Giancarlo Perrone & Anna M. Puglia \\
\hline Keisuke Miyauchi & Kara L. Nelson & Ogi Okwumabua & Everett Pesci & María J. Pujalte \\
\hline Ivona Mladineo & Tiffanie Nelson & Antonio Oliver & Gabriella Pessi & Katja Pulkkinen \\
\hline Luke A. Moe & Camilla Nesbø & Frank Oliver & Michael Pester & Anil K. Puniya \\
\hline William M. Moe & Markus Nett & Haley F. Oliver & John W. Peters & Maureen K. Purcell \\
\hline Ralf Moeller & Thomas R. Neu & James D. Oliver & Joseph E. Peters & Markus Puschenreiter \\
\hline Yvan Moënne-Loccoz & Josh D. Neufeld & Michael Olson & Scott Peterson & Meng Qi \\
\hline Sylvain Moineau & Kelly P. Nevin & Andrea M. & Snow B. Peterson & Dongru Qiu \\
\hline Pia Moisander & Elke Nevoigt & Olvera-Ramírez & Giulio Petroni & Huan Qiu \\
\hline Douwe Molenaar & Charles J. Newbold & Marc Ongena & Nicola K. Petty & John Quensen \\
\hline Alok K. Mondal & Diane G. Newell & Maarja Öpik & Stacy L. Pfaller & Amparo Querol \\
\hline Vicente Monedero & Dianne K. Newman & Anne Oppliger & Blaine Pfeifer & Jennifer J. Quinlan \\
\hline Christophe Monnet & Laura Newsome & Aharon Oren & Brian F. Pfleger & John P. Quinn \\
\hline Veronique Monnet & Irene L. Newton & Sarah A. Orlofske & Carol Phillips & Sandra Quinteira \\
\hline Josselin Montarry & Ryan J. Newton & William Orsi & Le T. Phung & Korneel Rabaey \\
\hline Rose A. Monteiro & Peter Nichols & Almudena Ortiz- & Aude Picard & Donato A. Raele \\
\hline Thomas J. Montville & Cheryl A. Nickerson & Urquiza & Birgit Piechulla & Petros I. Rafailidis \\
\hline Robert J. Moore & Kenneth Nickerson & Daniel O'Sullivan & David Pignol & Alok Raghav \\
\hline Sean D. Moore & Bernd Nidetzky & Isabelle P. Oswald & Suresh D. Pillai & Stephen W. Ragsdale \\
\hline Gabriel Mootian & Walter G. Niehaus & George O’Toole & Ameet J. Pinto & Ramya Rajagopalan \\
\hline Diego Mora & Alex T. Nielsen & Elizabeth Ottensen & Onruthai Pinyakong & S. Ramaswamy \\
\hline Pilar Morales & Dennis Nielsen & Soufian Ouchane & James M. Pipas & Manuel Ramírez \\
\hline Rocio Morales-Rayas & Jens Nielsen & Jia Ouyang & Christian Pirk & Christopher Rao \\
\hline Nancy Moran & Kaare M. Nielsen & Will A. Overholt & John M. Pisciotta & Brian H. Raphael \\
\hline
\end{tabular}


Lutgarde Raskin

David A. Rasko

Peter Rasmussen

Vipin K. Rastogi

Adam J. Ratner

Joerg Rau

Marina Rautenbach

Rumiana V. Ray

Raul R. Raya

Daniel S. Read

Sylvie Rebuffat

Karen B. Register

Stephen A. Rehner

Nicola M. Reid

Richard J. Reid-Smith

Georg H. Reischer

Cong Ren

Christopher Rensing

Martijn Rep

Tiina Reponen

Justus Reunanen

Federico Rey

Pascal Rey

Joon Haeng Rhee

Sung-Keun Rhee

Katharina Ribbeck

Bergmann M. Ribeiro

Maria L. Ricci

Eugene W. Rice

Scott A. Rice

Peter Richard

Gary P. Richards

Laurie Richardson

Susan E. Richardson

Hans H. Richnow

Stefan Riedel

Lasse Riemann

Lionel Rigottier-Gois

Thomas V. Riley

Rita V. Rio

Steven Ripp

David Ritchie

Kim Ritchie

Joseph Roberts

Courtney J. Robinson

D. A. Robinson

Eric Roden

Claudina

Rodrigues-Pousada

Susan B. Rodriguez

David

Rodríguez-Lázaro

Andrew Roe

David Rogers

Marko Rohlfs

Michel Rohmer

Thomas Rohrlack

Forest Rohwer

Thore Rohwerder

Fernando Rojo
Jesús L. Romalde

Manuel Romero

Alejandro Rooney

Diana Roopchanda

Ana Roque

Christine G. Roques

Jason W. Rosch

Philip C. Rosenstiel

Kimberly A. Ross

Silvia Rossbach

Amelia-Elena Rotaru

John R. Roth

Michael Rother

Mahmoud Rouabhia

Simon Roux

Denis Roy

Piklu Roy Chowdhury

Patricia Ruas-Madiedo

Luis A. Rubio

Knut Rudi

S. Emil Ruff

Stefan Ruhl

Françoise Rul

Jacob A. Russell

Una M. Ryan

Ivan Rychlik

Choong-Min Ryu

Sangryeol Ryu

Anita Rywinska

Zakee L. Sabree

Mourad Sabri

Ivan Sache

Elina Säde

Kazuhiko Saeki

Yuichi Saeki

Yolanda Sáenz

Elke Saile

Joelle K. Salazar

Hassan Salem

Irene Salinas

Hanna Salman

Siba K. Samal

John Samelis

José P. Sampaio

Buck Samuel

Scott Samuels

Gloria Sanchez

Mats Sandgren

Mario

Sandoval-Calderón

Carol H. Sandt

Dominique Sanglard

Daisuke Sano

Javier Santander

Eduardo Santero

Tasha M.

Santiago-Rodriguez

Sofia M. Santillana

Farakos
Jorge W. Santo

Domingo

Jesús A. Santos

Prakash K. Sarangi

Frank Sargent

Per E. Saris

Alain Sarniguet

Takashi Sasaki

Brandon Satinsky

Rohan Satishkumar

Dan Sato

Reetta Satokari

Karin Sauer

Michael Sauer

Kirsi Savijoki

Savvas Savvides

Dave J. Scanlan

Till F. Schäberle

Christopher W. Schadt

Amy Schaefer

Isabelle Schalk

Michael Scharf

Herb E. Schellhorn

Mark A. Schembri

Kirstin Scherlach

Peter Schierack

Dieter M. Schifferli

Jonathan S. Schilling

Axel Schippers

Michael Schlömann

John W. Schmidt

M. Alexander Schmidt

Michael G. Schmidt

Manfred J. Schmitt

Philippe

Schmitt-Kopplin

Catherine Schouler

Andreas Schramm

Matthew Schrenk

Volker Schroeckh

Albrecht Schulte-

Hostedde

Frederik Schulz

Stefan Schulz

Julia Schumacher

Markus Schuppler

Michael J. Schurr

Gerrit J. Schut

Egbert Schwartz

Matthew Scotch

H. Morgan Scott

R. B. Seale

Sarah C. Seaton

Anna M. Seekatz

Harald Seifert

Yuji Sekiguchi

David A. Sela

Thorsten Selmer

John M. Senko

Jin-Ho Seo
Diana I. Serrazanetti

Alain L. Servin

Luis E.

Servín-Garcidueñas

Luca Settanni

Konstantin V.

Severinov

Ashley Shade

Devendra H. Shah

Robert M. Shanks

Jiahui Shao

Zongze Shao

Lori R. Shapiro

James P. Shapleigh

Nikki Shariat

Manan Sharma

Vijay K. Sharma

Thomas Sharpton

Evgenya S. Shelobolina

Yulong Shen

Louis A. Sherman

Emma Sherwood

Vladimir Shevchik

Liang Shi

Joseph Shiloach

Motoyuki Shimizu

Masaki Shintani

A. Sibirny

Steven D. Siciliano

Johannes Sikorski

Roberto Silva

Simon D. Silver

Jerry Simecka

Christopher Simmons

Manuel Simões

Holly M. Simon

Rahul Singh

Naresh Singhal

Albert Siryaporn

Kaarina Sivonen

James M. Slauch

Joan L. Slonczewski

Melanie Smee

Ronald D. Smiley

Colin P. Smith

Kerry S. Smith

Robert P. Smith

S. L. Smith

Stuart C. Smith

Theresa J. Smith

Val H. Smith

Evan S. Snitkin

Jamie C. Snyder

Dmitri Sobolev

Pablo Sobrado

Marc Solioz

Katie Solomon

Alicia Solorzano

Bongkeun Song

Lirong Song

Anton Sonnenberg

Sebastian R. Sorensen

Uffe B. Sørensen

Joseph A. Sorg

Mark R. Sosnowski

Sonia A.

Soto-Rodriguez

Ana Sousa

Jim C. Spain

Giuseppe Spano

Vanessa Sperandio

Daan R. Speth

Richard Splivallo

Georg Sprenger

Dirk Springael

Srinand Sreevatsan

Gary Stacey

Christopher Staley

Boris U. Stambuk

Kim Stanford

Thad B. Stanton

Jan Steensels

Christian Steinberg

Laurey Steinke

Howard M. Steinman

Eike Steinmann

Drake C. Stenger

Roy H. Stevens

Bradley S. Stevenson

Frank Stewart

Philip E. Stewart

Valley Stewart

O. C. Stine

Alain C. Stintzi

Virginia O. Stockwell

Andreas Stolz

Paul Stoodley

Paul D. Straight

Michael R. Strand

Laura K. Strawn

Marc Strous

David Stuart

Jörg Stülke

Gregory Sturbaum

Hikaru Suenaga

Masayuki Sugawara

Irshad M. Sulaiman

Tarah Sullivan

Anne O. Summers

Xingmin Sun 


\begin{tabular}{|c|c|c|c|c|}
\hline Eduardo N. Taboada & Gaku Tokuda & Martha Vaughan & Yong Wang & Michael Wisniewski \\
\hline John R. Tagg & Bradley B. Tolar & Anthony G. & Michael Ward & Verena Witt \\
\hline Kapil Tahlan & Snezana Tomanovic & Vecchiarelli & D. Warnecke & Thomas E. Wittum \\
\hline Martial Taillefert & Francisco & Fernando Vega & Jeffrey M. Warner & Dagmar Woebken \\
\hline Teruhiro Takabe & Tomás-Barberán & Tatiana B. Venkova- & Martin J. Warren & Mark J. Wolcott \\
\hline Masayuki Takahashi & Giuseppina & Canova & Kazuya Watanabe & Amanda Wollenberg \\
\hline Yurika Takahashi & Tommonaro & Mario A. Vera & Boris Wawrik & Elisha M. Wood- \\
\hline Daisuke Takamatsu & Thomas A. Tompkins & Cornel Verduyn & Andrew L. Webb & Charlson \\
\hline Yuichi Takikawa & Edward Topp & Tom F. Verhaeghe & Carolyn F. Weber & Mhairi Workman \\
\hline Guylaine Talbot & César I. Torres & Niels O. Verhulst & Hongping Wei & Han A. Wösten \\
\hline Paula Tamagnini & Sandra Torriani & Christian Verniére & Huang Wei & Tanja Woyke \\
\hline Hideyuki Tamaki & Maria C. Touz & Kevin Verstrepen & Wei Wei & Anita C. Wright \\
\hline Rita Tamayo & Masanori Toyofuku & Marta Veses-Garcia & Jennifer L. Weidhaas & Gerard D. Wright \\
\hline Cecilia Tamborindeguy & Cory G. Toyota & Stephen J. Vesper & Paul J. Weimer & Kelly Wrighton \\
\hline Huarong Tan & Lindsay Triplett & José M. Viader-Salvadó & Markus G. Weinbauer & Danielle Wroblewski \\
\hline Bing Tang & Marion Tseng & Jorge E. Vidal & Bevan S. Weir & Bian Wu \\
\hline Xiao-Feng Tang & Seiji Tsuge & Ene Viiard & Bernard Weisblum & Hui Wu \\
\hline Yi-Wei Tang & Takeharu Tsuge & Erik Vijgenboom & David S. Weiss & Kai Wu \\
\hline Valter Tanodi & Bettina Tudzynski & Laura Villanueva & Laure Weisskopf & Lingling $\mathrm{Wu}$ \\
\hline Silvana Tarlera & Burkhard Tuemmler & Eric N. Villegas & Roy D. Welch & Longfei Wu \\
\hline Neslihan Tas & Raymond J. Turner & Isabelle Villena & David F. Welche & Weimin Wu \\
\hline Taurai Tasara & Alessandra Turrini & Jose M. Vinardell & Martin Welker & Markus Wyss \\
\hline Yosuke Tashiro & Francesca Turroni & Boris A. Vinatzer & David M. Weller & Song Xiang \\
\hline Andreas Tauch & Silvia Turroni & Jan Vinjé & Derek H. Wells & Xiang Xiao \\
\hline Michael E. Taveirne & Kenji Ueda & Eszter Virág & Jim Wells & Jingjing Xie \\
\hline Michael W. Taylor & Gaylen A. Uhlich & Marie-Joelle Virolle & Jia Wen & Jun-Feng Xie \\
\hline Neung Teaumroong & Matthias S. Ullrich & Karen L. Visick & -hua Wen & $\mathrm{g} \mathrm{Xu}$ \\
\hline Christoph C. Tebbe & Can M. Ünal & Jorge M. Vivanco & Zezhang T. Wen & Peng Xu \\
\hline Stephen M. Techtmann & Dave W. Ussery & Martin Von Bergen & David Werner & Tao Xu \\
\hline Lucia M. Teixeira & Mieke Uyttendaele & Erik Von Rosenvinge & Brian Werth & Wang Xuan \\
\hline Miguel C. Teixeira & Nathalie Vachiery & Christian R. Voolstra & David J. Westenberg & Yadav \\
\hline Ben Temperton & Juan J. Valdez-Alarcón & Jamie Voyles & Benita & Michail Yakimov \\
\hline Leonard M. Tender & Alexandra Valencakova & Carmen Wacher & Westerlund-Wikström & Toshiharu Yakushi \\
\hline Max Teplitski & Minoska Valli & Masaaki Wachi & Janet Westpheling & Yoshihisa Yamashita \\
\hline Akihiko Terada & Miguel A. Valvano & Wagner & Whitby & Jun Yan \\
\hline Tohru Terada & Angel Valverde & Irene Wagner-Döbler & Cynthia B. Whitchurch & Yajun Yan \\
\hline Benno H. ter Kuile & Heleen Van Acker & David W. Waite & Chris A. Whitehouse & Chen Yang \\
\hline Rudolf K. Thauer & Willem J. van Berkel & Takayoshi Wakagi & Marvin Whiteley & Ching-Hong Yang \\
\hline Elitza S. Theel & Ineke K. Van Boeijen & Samantha Wales & William B. Whitman & Fan S. Yang \\
\hline Kevin R. Theis & Anne M. van der Does & Seth T. Walk & Jason K. Whitmire & Min Yang \\
\hline Casey M. Theriot & Wilfred A. van der Donk & Alan W. Walker & Brooke M. Whitney & Ruifu Yang \\
\hline Johan M. Thevelein & Dick van der Kooij & Anne-Sophie Walker & hyte & Sizhong Yang \\
\hline Chris M. Thomas & George van der Merwe & Michelle E. Walker & Reed B. Wickner & Yang Yang \\
\hline Torsten Thomas & Paul W. van der Wielen & Virginia K. Walker & Fritz Widdel & Yi-Ting Yang \\
\hline Vinai C. Thomas & Jonathan D. Van & Daniel Wall & Wiedmann & Zhaomin Yang \\
\hline Vincent Thomas & Hamme & John Wallace & n K. Wiegel & Anthony C. Yannare \\
\hline Linda S. Thomashow & Rob Van Houdt & David Walsh & Gregory D. Wiens & Emi Yasuda \\
\hline R. C. Andrew & Jan van Kan & Jens Walter & Krista R. Wigginton & Marylynn V. Yates \\
\hline Thompson & Geertje van Keulen & Aijie Wang & Reto S. Wijker & Yutaka Yawata \\
\hline Jon Thorson & Ed W. van Niel & Dongbo Wang & Siouxsie Wiles & Chris Yeager \\
\hline J. Cameron Thrash & Karl-Heinz van Pee & Dongping Wang & Brian J. Wilkinson & Nathan Yee \\
\hline Rebecca Thurber & Jan P. van Pijkeren & Fengping Wang & Rob J. Willems & Alexis P. Yelton \\
\hline Chang F. Tian & Vicky L. van Santen & Gejiao Wang & Kenneth Williams & Carl Yeoman \\
\hline Peng Tian & Douwe van Sinderen & Guangyi Wang & Stanly Williams & Fitnat H. Yildiz \\
\hline Ming Tien & Arnoud H. M. van Vliet & Haifeng Wang & Thomas Williams & Christopher K. Yost \\
\hline Peter Tijssen & Gilles P. van Wezel & Hua H. Wang & Tiffany Williams & Lingchong You \\
\hline Peer H. Timmers & Leonardo J. van Zyl & Hui Wang & Paul Wilmes & Kevin D. Young \\
\hline Louis S. Tisa & Cristian Varela & Linqi Wang & Stephen C. Winans & Vincent Young \\
\hline Dirk Tischler & Vitor Vasconcelos & Qiuhong Wang & Klaus Winzer & Loubna Youssar \\
\hline Laurent Toffin & Joachim Vater & Shihua Wang & Margaret C. Wirth & $\mathrm{Bo} \mathrm{Yu}$ \\
\hline
\end{tabular}


Jae-Hyuk Yu

Ri-Qing Yu

Zhongtang Yu

Joshua S. Yuan

Zhiguo Yuan

Ulrich Zahringer

Jindong Zan

Regis A. Zanette

Davide Zannoni
Giacomo Zara
Severino Zara
Robert Zarnowski
Raffaele Zarrilli
Einat Zchori-Fein
Takeshi Zendo
Quan Zeng

Josef Zeyer

Buchang Zhang

Cheng-Cai Zhang

Guochang Zhang

Kechun Zhang

Li-Mei Zhang

Li-Qun Zhang

Shuping Zhang

Tong Zhang
Wen Zhang
Yan Zhang
Jiangchao Zhao
Ming-Wen Zhao
Xin Zhao
Yang Zhao
Jie Zheng

Yong Zheng Jian-Jiang Zhong Jingwen Zhou Zhihua Zhou Jiangfeng Zhu Julie L. Zilles Stephen H. Zinder Barry Ziola 